\title{
Cerebral Infarction from Compression of the Internal Carotid Artery - A Rare Presentation of Pituitary Apoplexy
}

\author{
Kali Uppalapati", Narindra Ramnarine, FRCS and Panduranga Seetahal-Maraj \\ Department of Neurosurgery, San Fernando General Hospital, Trinidad and Tobago, West Indies
}

*Corresponding author: Kali Uppalapati, Department of Neurosurgery, San Fernando General Hospital, 7A Mon Repos Heights, Cascade, Trinidad and Tobago, West Indies, Tel: (+1)-868-376-6054

\begin{abstract}
Background and importance: Cerebral infarction caused by Pituitary Apoplexy is a rare phenomenon with less than 30 reported cases in literature. We report this rare phenomenon in a 42-year-old female, who was diagnosed and underwent urgent trans-sphenoidal decompression of the sella.

Clinical presentation: A 42-year-old female presented with sudden onset of headaches, left hemiplegia and bitemporal hemianopia. Radiological investigations revealed right anterior circulation infarction from mechanical obstruction of the right internal carotid artery by Pituitary Apoplexy. She underwent an urgent transsphenoidal decompression of the sella and had gradual resolution of symptoms.

Conclusion: Mechanical compression of the cerebral vasculature and vasospasm are proposed to be the two main mechanisms of infarction. This atypical cause of cerebral ischemia should be considered in any patient with a pituitary adenoma, it should also be added to the list of etiologies of cerebrovascular accidents. There is no consensus on the ideal timing of surgery, due to limited case reports in the literature. Currently, urgent transsphenoidal decompression is a proven method of treatment, and can lead to good outcomes in properly selected patients.
\end{abstract}

\section{Keywords}

Pituitary apoplexy, Cerebral infarction, Pituitary adenoma, ICA compression, Stroke

\section{Abbreviations}

PA: Pituitary Apoplexy; TSR: Transsphenoidal Resection; CT: Computed Topography; MRI: Magnetic Resonance Imaging; MRC: Medical Research Council; SAH: Subarachnoid Haemorrhage; ICA: Internal Carotid Artery; ACA: Anterior Cerebral Artery; MCA: Middle Cerebral Artery

\section{Introduction}

Pituitary apoplexy is an uncommon but potentially life-threatening complication of pituitary adenomas. The clinical syndrome can include the acute onset of severe headaches, visual deficits, cranial nerve palsies and partial/total endocrine dysfunction. It is due to either infarction or hemorrhage within a pituitary adenoma and can lead to significant morbidity and even mortality. Not all patients present with the classically described symptomology of this syndrome. It is prudent to be aware of the variable clinical manifestations of this condition.

We report on an unusually rare presentation of pituitary apoplexy, in a patient with both clinical and radiological features of right internal carotid artery infarction. This was from mechanical compression of the vessel by expansion of an apoplectic pituitary adenoma. The patient required urgent neurosurgical intervention to prevent life-threatening complications.

\section{Case Report}

A 42-year-old female presented to the emergency department with complaints of headaches and vomiting for 2 days duration, and acute onset of left sided weakness for 6 hours. She denied any seizures, fever, or visual deficits. She had no known comorbidities, and there were no significant positives in the rest of the history.

On physical examination, she was hemodynamically stable, with no evidence of an Addisonian crisis. Her Glasgow coma scale (GCS) was 15/15 and pupils were

Citation: Uppalapati K, Ramnarine N, Seetahal-Maraj P (2021) Cerebral Infarction from Compression of the Internal Carotid Artery - A Rare Presentation of Pituitary Apoplexy. Neurosurg Cases Rev 4:080. doi.org/10.23937/2643-4474/1710080

Accepted: September 18, 2021; Published: September 20, 2021

Copyright: (c) 2021 Uppalapati K, et al. This is an open-access article distributed under the terms of the Creative Commons Attribution License, which permits unrestricted use, distribution, and reproduction in any medium, provided the original author and source are credited. 
$3 \mathrm{~mm}$ and reactive to light bilaterally. Visual acuity was preserved and there no evidence of ophthalmoplegia. Tone was noted to be increased in the left upper and lower limbs. Power was graded as $5 / 5$ (MRC) on the entire right side, whereas it was $0 / 5$ on the left side. She was hyper-reflexic on the left, with upgoing left plantar reflex.

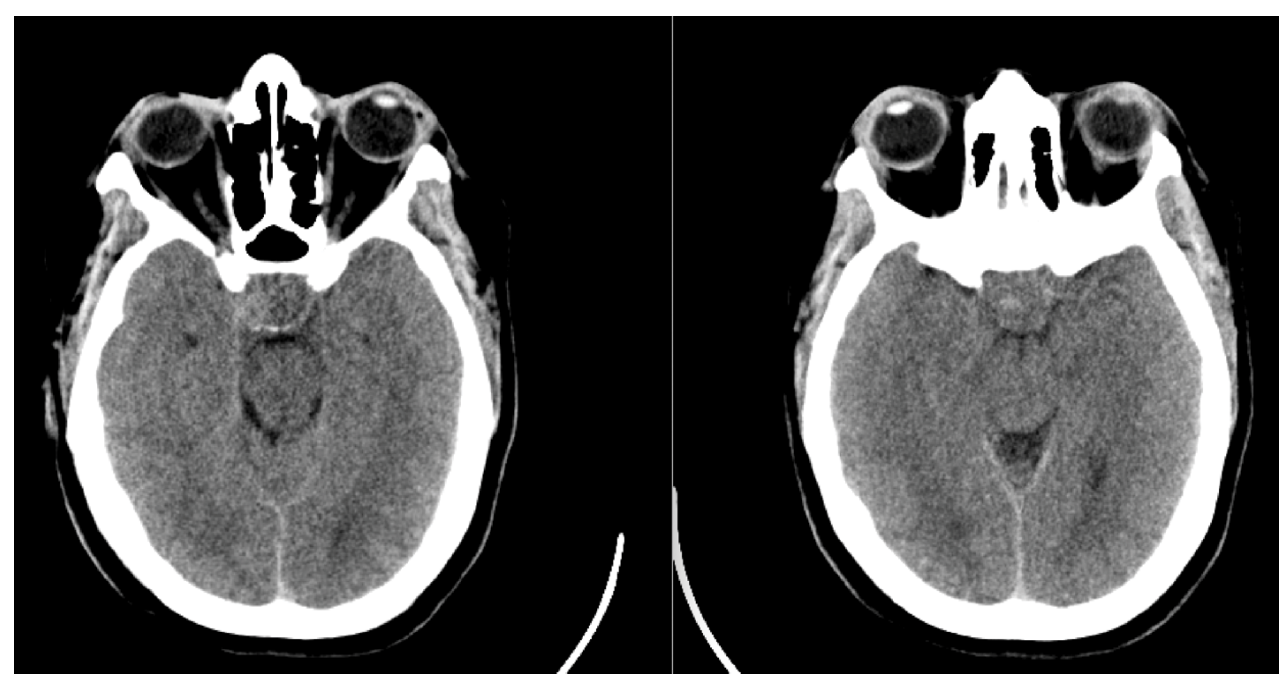

Figure 1: Non-contrast CT scan of the brain on presentation, showing a heterogenous mass in the sella with later extension in the right cavernous sinus.

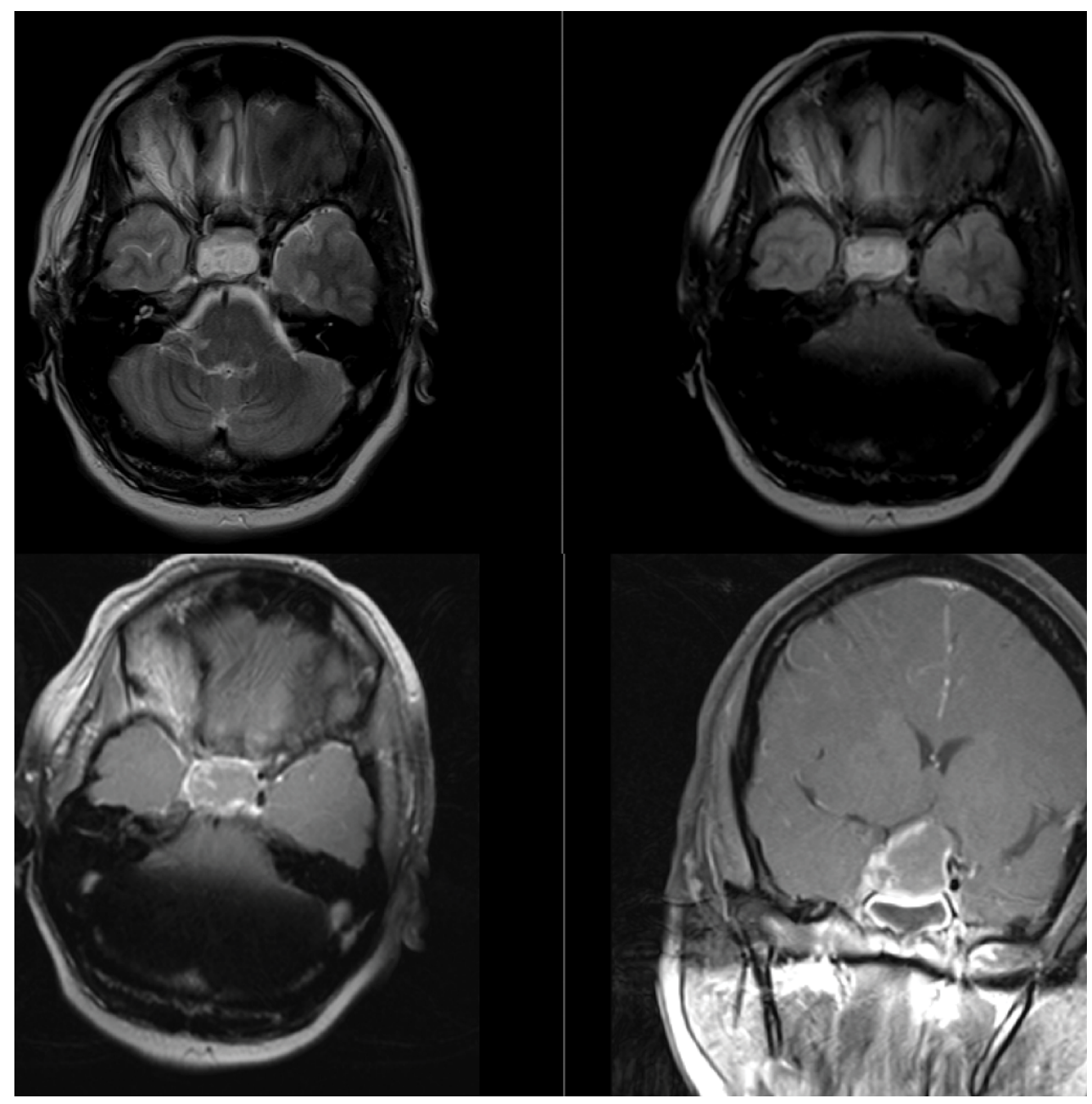

Figure 2: Top left: T2 weighted axial image showing heterogenous signal intensity within the sella with right lateral extension into the cavernous sinus and compression at the supraclinoid segment of the right ICA; Top right: T1 pre contrast image showing high signal intensity within the pituitary gland suggesting an apoplexy. Bottom left and right: Axial and Coronal planes of T1 post contrast images showing a large ovoid mass with minimal enhancement with expansion into the suprasellar cistern displacing the optic chiasma minimally. The apoplectic adenoma is expanding laterally into the cavernous sinus with direct compression at the supraclinoid segment of the right. 
CT scan of the brain showed a hypodense region in the territory of the right ACA and MCA, consistent with an acute ischemic infarct (Figure 1), but no significant mass effect or midline shift. A hyperdense mass was noted in the region of the pituitary fossa, suspicious for a hemorrhage in a pituitary adenoma. The patient had an urgent MRI/MRA of the brain, which revealedanapoplectic grade 3 pituitary adenoma. There was compression of the cavernous segment of the right ICA (Figure 2), which was the likely culprit for her ischaemic infarct.

The patient was admitted to the neurosurgical service for optimization and definitive treatment. Pituitary hormone panel showed a mild decrease in prolactin, T3 and TSH, with the remainder within normal ranges. Formal visual field testing was done, and revealed bitemporal hemianopia, consistent with optic chiasm compression. Steroids were commenced, and preparation made for surgical decompression.

However, the patient had an acute reduction in her GCS to $9 / 15$. Repeat CT brain showed maturation of the right ICA infarct, but no mass effect or midline shift. There was further lateral expansion of the haematoma from the apoplectic adenoma. Consent was obtained for an urgent transsphenoidal decompression of the pituitary adenoma via endoscopic-assisted microscopic approach.

The sella was decompressed, and tissue samples were sent for histopathological evaluation. Intracranial micro-doppler assessment confirmed adequate flow through both ICAs. Immediate post-operative CT angiogram confirmed complete decompression of the sella, and restoration of the normal caliber and flow of the right ICA. (Figure 3 ).

The patient had an uneventful post-operative course, with gradual improvement in her strength on the left side. Repeat pituitary hormone panel showed mild reduction in TSH and T3, consistent with the preoperative profile. Final histological review confirmed a pituitary adenoma.

At 3-month follow up, repeat visual field testing revealed reversal of the previous bitemporal hemianopia. Motor exam findings included grade $3 / 5$ power in the left upper and lower limbs. There were no symptoms of pituitary dysfunction. Serum hormone levels were within normal limits.

\section{Discussion}

Pituitary apoplexy is an uncommon clinical syndrome in patients with pituitary adenomas. It has a quoted incidence of $0.6-9 \%$ in the existing literature $[1,2]$. Its aetiology is either haemorrhage or infarction within the adenoma. Patients can present with varying signs and symptoms, most commonly including headaches, nausea, diminished visual acuity, temporal visual field cut, ophthalmoparesis and impaired mental status [15]. Risk factors for PA includes bromocriptine withdrawl or initiation, head trauma, intracranial hypertension, hormone stimulation of the gland (for example during pregnancy), large tumor, cavernous sinus invasion and anticoagulation $[4,5]$.

\section{Cerebral ischaemia due to PA}

Cerebral ischaemia and infarction due to PA is an extremely rare phenomenon. The current literature contains 29 case reports on this syndrome [2,6]. The proposed mechanisms behind cerebral infarction from PA are two-fold. One theory is from mechanical obstruction of the ICA by an abrupt expansion of the adenoma. The second is vasospasm of the vessels around the sella from the subarachnoid hemorrhage (SAH). The apoplectic tumor secrets vasoactive substance which then enters the subarachnoind space and causes vasospams. Some authors believe both mechanisms are responsible $[2,3,6]$.

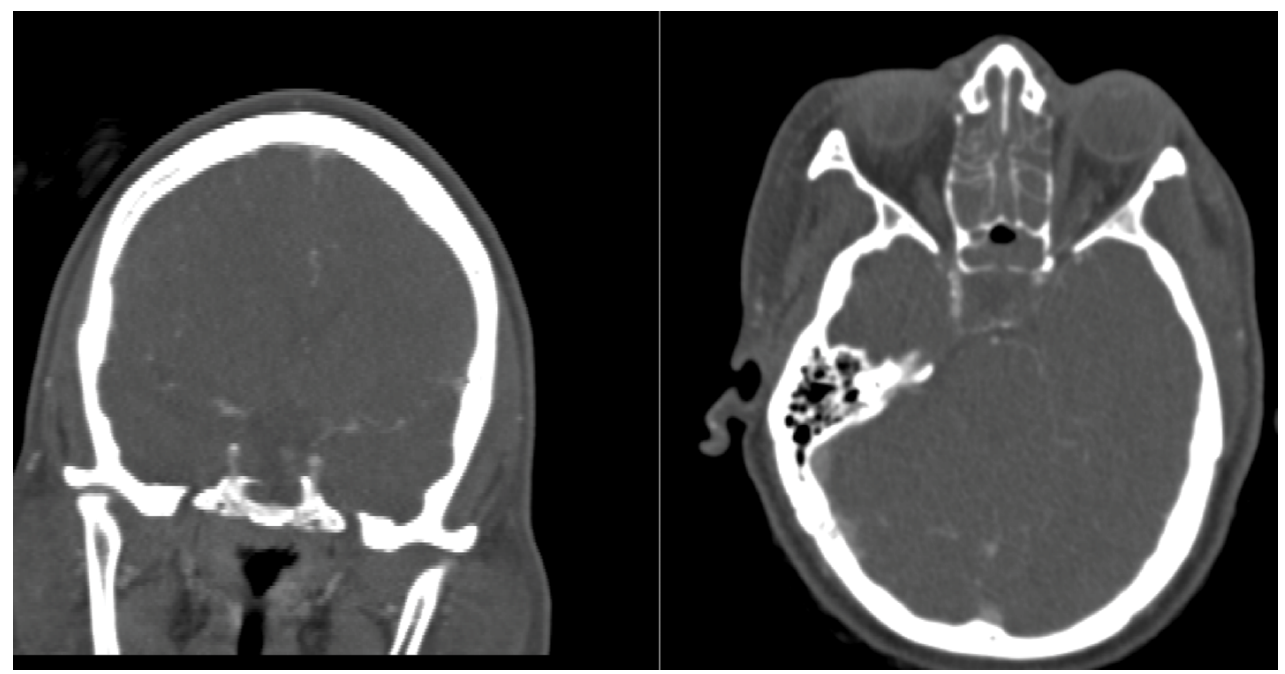

Figure 3: Coronal and Axial images of CT angiogram done post operatively showing restored flow through the right internal carotid artery. 
The most affected artery is the ICA followed by the ACA, MCA and basilar arteries [3,4].

In our case, there was radiological evidence of intratumoral hemorrhage resulting in the compression and stenosis of the supraclinoidal segment of the right ICA due to mechanical forces exerted on the vessel (Figure 2). This resulted in the right-sided anterior circulation cerebral infarct and left hemiplegia. Rapid expansion from the hemorrhage results in raised intrasellar pressure of up to $58 \mathrm{mmHg}$ in PA. This results in lateral displacement of the adenoma into the cavernous sinus and subsequent compression of the ICAs which results in the cerebral ischemia [7].

\section{Diagnosis}

The diagnosis of PA is made by either CT or MRI of the brain, which can reveal haemorrhage within the sella. On $\mathrm{CT}$, the adenoma may not be clearly visualized, therefore MRI with pituitary protocol is necessary in many cases. One series comparing sensitivity of radiologic investigations in PA found that CT scans identified only $21 \%$ of pituitary tumour haemorrhages, compared to an $88 \%$ detection rate on MRI [8]. In patients with cerebral ischaemia, CT or $\mathrm{MR}$ angiograms can evaluate for stenosis or compression of the ICA from PA [3]. Confirmatory imaging with a cerebral catheter angiogram can also be useful to differentiate between vasospasm and compression, it is thought that there is bilateral involvement of cerebral arteries in vasospasm $[3,4]$.

\section{Management}

There is extensive evidence in advocating an early decompression surgery in patients with visual symptoms or endocrine dysfunction associated with PA. There is evidence of better outcomes with early surgery in these cases $[5,9]$. The optimal timing of surgery has not been determined [10] but it is divided as early (within 7 days of presentation) and delayed [4,7]. The goal of decompressive surgery is to reduce intrasellar pressure and improve cerebral perfusion. This aids in prevention of further neurologic deterioration. In our case, a decision for early surgery was made to minimize the morbidity that results from subsequent cerebral infarction. This would allow rapid decompression of the ICA, reversal of stenosis and improved cerebral perfusion.

\section{Outcomes}

Cerebral infarction associated with PA involves major cerebral vessels, the morbidity and mortality can be substantial. In a review by Jiang, et al. in 2020, the mortality rate was $26.8 \%$ [3]. In comparison, the mortality of PA alone ranges from $0-12.5 \%[11,12]$. The cause of death in patients with PA and cerebral ischemia is as a result of intracranial hypertension and neuronal death.

\section{Conclusion}

PApresenting with cerebral infarction is a rare occurrence, with only sporadic reports in the literature. Mechanical compression of the cerebral vasculature and vasospasm are proposed to be the two main mechanisms of infarction. This atypical cause of cerebral ischemia should be considered in any patient with a pituitary adenoma, it should also be added to the list of etiologies of cerebrovascular accidents. There is no consensus on the ideal timing of surgery, due to limited case reports in the literature. Standardised reporting of these cases is recommended to allow detailed analysis which would aid in development of protocols to treat this condition. Currently, transsphenoidal decompression is a proven method of treatment, and can lead to good outcomes in properly selected patients.

\section{Authors Declaration}

All Authors have contributed equally to the creation, drafting and editing of this manuscript. There were no sources of funding and none of the authors have financial disclosures.

\section{References}

1. Zou Z, Liu C, Sun B, Chen C, Xiong W, et al. (2015) Surgical treatment of pituitary apoplexy in association with hemispheric infarction. J Clin Neurosci 22: 1550-1554.

2. Elarjani T, Chen S, Cajigas I, Saway B, Sur S, et al. (2020) Pituitary apoplexy and cerebral infarction: Case report and literature review. World Neurosurg 141: 73-80.

3. Jiang Q, Xiao S, Shu L, Huang X, Chen X, et al. (2020) Pituitary apoplexy leading to cerebral infarction: A systematic review. Eur Neurol 83: 121-130.

4. Van Dong H, Tran D, Chu HT, Pham AH, Nguyen XT, et al. (2021) Emergency endoscopic surgery for pituitary apoplexy presenting as cerebral infarction in a limited resources condition: A case report. Int $\mathrm{J}$ Surg Case Rep 83: 106015.

5. Bi WL, Dunn IF, Laws ER (2015) Pituitary apoplexy. Endocrine Reviews 69-75.

6. Banerjee C, Snelling B, Hanft S, Komotar RJ (2015) Bilateral cerebral infarction in the setting of pituitary apoplexy: A case presentation and literature review. Pituitary 18: 352-358.

7. Pokhrel B, Khanal S, Chapagain P, Sedain G (2021) Pituitary apoplexy complicated by cerebral infarction: A case report. JNMA J Nepal Med Assoc 59: 723-726.

8. Randeva HS, Schoebel J, Byrne J, Esiri M, Adams CBT, et al. (1999) Classical pituitary apoplexy: Clinical features, management and outcome. Clin Endocrinol (Oxf) 51: 181188.

9. Bills DC, Meyer FB, Laws ERJ, Davis DH, Ebersold MJ, et al. (1993) A retrospective analysis of pituitary apoplexy. Neurosurgery 33: 602-609.

10. Chokyu I, Tsuyuguchi N, Goto T, Chokyu K, Chokyu M, et al. (2011) Pituitary apoplexy causing internal carotid artery occlusion. Neurol Med Chir (Tokyo) 51: 48-51.

11. Bujawansa S, Thondam SK, Steele C, Cuthbertson DJ, Gilkes CE, et al. (2014) Presentation, management and outcomes in acute pituitary apoplexy: A large single-centre experience from the United Kingdom. Clin Endocrinol (Oxf) 80: 419-424.

12. Dubuisson AS, Beckers A, Stevenaert A (2007) Classical pituitary tumour apoplexy: Clinical features, management and outcomes in a series of 24 patients. Clin Neurol Neurosurg 109: 63-70. 